Raum und Zeit 
Erlebe Schönes und vergiss die Zeit. 
Andreas Müller

\section{Raum und Zeit}

Vom Weltall zu den Extradimensionen von der Sanduhr zum Spinschaum 
Dr. Andreas Müller

Technische Universität München

Exzellenzcluster Universe

Boltzmannstr. 2

85748 Garching

ISBN 978-3-8274-2858-5

Die Deutsche Nationalbibliothek verzeichnet diese Publikation in der Deutschen Nationalbibliografie; detaillierte bibliografische Daten sind im Internet über http://dnb.d-nb.de abrufbar.

Springer Spektrum

(C) Springer-Verlag Berlin Heidelberg 2013

Das Werk einschließlich aller seiner Teile ist urheberrechtlich geschützt. Jede Verwertung, die nicht ausdrücklich vom Urheberrechtsgesetz zugelassen ist, bedarf der vorherigen Zustimmung des Verlags. Das gilt insbesondere für Vervielfältigungen, Bearbeitungen, Übersetzungen, Mikroverfilmungen und die Einspeicherung und Verarbeitung in elektronischen Systemen.

Die Wiedergabe von Gebrauchsnamen, Handelsnamen, Warenbezeichnungen usw. in diesem Werk berechtigt auch ohne besondere Kennzeichnung nicht zu der Annahme, dass solche Namen im Sinne der Warenzeichen- und Markenschutz-Gesetzgebung als frei zu betrachten wären und daher von jedermann benutzt werden dürften.

Planung und Lektorat: Vera Spillner, Stefanie Adam

Einbandabbildung: Hauptbild: (c) Mag. Dr. Werner Benger; Konrad-Zuse-Zentrum für Informationstechnik, Berlin; Max-Planck-Institut für Gravitationsphysik, Potsdam; Center for Computation \& Technology at Louisiana State University, USA; Institute for Astro- and Particle Physics at University of Innsbruck, Austria. Bildreihe links: C James Overduin, Pancho Eekels and Bob Kahn, Sanduhr: (C) Dmitry Rukhlenko - Fotolia.com, Bildreihe Mitte: Andreas Müller, Bildreihe rechts: NASA, ESA and J. Hester (Arizona State University)

Einbandentwurf: SpieszDesign, Neu-Ulm

Gedruckt auf säurefreiem und chlorfrei gebleichtem Papier

Springer Spektrum ist eine Marke von Springer DE. Springer DE ist Teil der Fachverlagsgruppe Springer Science+Business Media.

www.springer-spektrum.de 


\section{Vorwort}

Raum und Zeit scheinen uns so vertraut und selbstverständlich, dass wir uns gewöhnlich keine Gedanken darüber machen. Doch hat die Entwicklung der Physik gelehrt, dass es sich bei genauer Betrachtung ganz anders damit verhält, als die alltägliche Erfahrung suggeriert. Wie wir seit Einsteins „Wunderjahr“ 1905 wissen, existieren Raum und Zeit nicht absolut und für sich selbst, sondern bilden eine Einheit - die Raumzeit. Und zehn Jahre später erkannte er, dass die Raumzeit selbst am dynamischen Geschehen teilnimmt und sich aus ihrer Krümmung die Effekte der Schwerkraft geometrisch verstehen lassen. Auf einfachen Prinzipien beruhend, erklären die Einstein'schen Feldgleichungen alle mit der Gravitation zusammenhängenden Beobachtungen, von der Planetenbewegung bis zur Expansion des Universums nach dem Urknall. Und das so genau, dass bis heute keine Messung eine Abweichung feststellen konnte.

Dennoch deuten zahlreiche Hinweise auf einen bevorstehenden Umbruch der Physik. Trotz ihrer überwältigenden Erfolge sind die Einstein'sche Theorie, welche die Physik bei großen Abständen beschreibt, und die Quantenmechanik (bzw. die Quantenfeldtheorie), welche für die physikalischen Vorgängen im Kleinen verantwortlich ist, unvollständig und möglicherweise sogar inkonsistent - u. a. schon deshalb, weil die beiden Theorien in ihrer gegenwärtigen Form einfach nicht zusammenpassen wollen! Die Suche nach einer Theorie der Quantengravitation, welche beide zusammenführen 
und ihre inneren Widersprüche auflösen soll, ist so zur größten Herausforderung der theoretischen Physik geworden. Aber trotz einer kollektiven intellektuellen Anstrengung ohne Beispiel in der Geschichte der Physik, an der sich weltweit viele theoretische Physiker beteiligen, sind wir der ,richtigen“ Theorie bis jetzt kaum näher gekommen.

Das vorliegende Buch führt den Leser an die Vorderfront der aktuellen Forschung. Nach einem ausführlichen Streifzug durch die Geschichte der Physik von Raum und Zeit bis zu ihrer Vereinigung in der Raumzeit, bei dem auch neueste Erkenntnisse (wie z. B. Dunkle Energie und Gravitationswellen betreffend) zur Sprache kommen, wendet es sich einigen der Ansätze zu, welche heute bei der Suche nach der Quantengravitation verfolgt werden. Neben der Stringtheorie, dem vielleicht aussichtsreichsten Ansatz, gehören dazu u. a. die Schleifenquantengravitation und Modelle, welche die Existenz weiterer Dimensionen der Raumzeit postulieren. Die Herausforderung, deren z. T. sehr esoterisches Formelwerk in eine verständliche Sprache zu übersetzen, hat Andreas Müller hervorragend gemeistert. So wird der Leser viel Spaß an dieser „Momentaufnahme“ der aktuellen Forschung haben.

Hermann Nicolai

(Max-Planck-Institut für Gravitationsphysik, Golm) 


\section{Inhalt}

Vorwort $\ldots \ldots \ldots \ldots \ldots \ldots \ldots \ldots \ldots \ldots, \quad \mathrm{V}$

1 Einführung $\ldots \ldots \ldots \ldots \ldots \ldots \ldots \ldots \ldots \ldots \ldots \ldots \ldots \ldots$

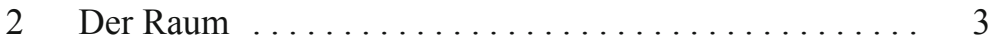

2.1 Raum im Alltag .................... 3

2.2 Raumkoordinaten und Raumskala ............. 4

2.3 Der Weltraum ....................... 9

2.4 Newtons absoluter Raum .................. 11

3 Die Zeit ........................... 19

3.1 Zeit im Alltag ...................... 19

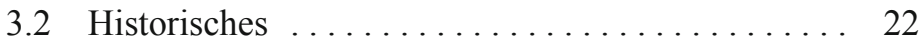

3.3 Zeitmessung und Uhren $\ldots \ldots \ldots \ldots \ldots \ldots \ldots . \ldots 24$

3.4 Blicke in die Vergangenheit ................. 39

3.5 Zeitpfeile ........................ 43

3.6 Blick zurück: Kosmos der Teilchen und Quanten ...................... 55

3.7 Blick nach vorn: Kalter, dunkler Kosmos . . . . . . 65

3.8 Galileis absolute Zeit . . . . . . . . . . . . . . 75

4 Die Raumzeit . ........................ 79

4.1 Licht und die Spezielle Relativitätstheorie ...... 79

4.2 Minkowskis flache, vierdimensionale Welt ..... 83 
4.3 Einsteins neue Gravitation: Allgemeine

Relativitätstheorie ................. 86

4.4 Zeit und Länge sind relativ . . . . . . . . . . . . 91

4.5 Die Raumzeit der Sonne und der Erde . . . . . . . . 96

4.6 Die Raumzeit kompakter Objekte . . . . . . . . . 105

4.7 Wellen der Raumzeit: Gravitationswellen .... . . . 108

4.8 Die Raumzeit des Universums . . . . . . . . . . . . . 111

4.9 Singularitäten der Raumzeit . . . . . . . . . . . 118

5 Das neue Wesen von Raum und Zeit? . . . . . . . . . . . . 125

5.1 Jenseits bewährter Theorien . . . . . . . . . . . . 125

5.2 Mehr Raum? Räumliche Extradimensionen .... . 128

5.3 Gibt es Längen- oder Zeitquanten? . . . . . . . . . 134

5.4 Ausblick auf die Forschung . . . . . . . . . . . 142

6 Gedanken zum Schluss . . . . . . . . . . . . . 151

6.1 Was war vor dem Urknall? . . . . . . . . . . . . 151

6.2 Auf der Spur nach dem Wesen von Raum und Zeit . . . . . . . . . . . . . . . . . . . . . 160

6.3 Die drei Verständnisebenen für Raum

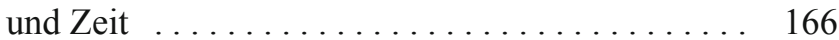

Glossar ......................... 169

Abbildungsverzeichnis ................ 185

Danksagungen ... . . . . . . . . . . . . . . . . . 193

Quellen und weitere Literatur . . . . . . . . . . . . . 195

Index . . . . . . . . . . . . . . . . . . . 199 